\title{
Anatomia de escapos de Tillandsia L. (Bromeliaceae) dos Campos Gerais do Paraná, Brasil ${ }^{1}$
}

\author{
SIMONE SEGECIN ${ }^{2,4}$ e VERA L. SCATENA ${ }^{3}$
}

(recebido: 2 de maio de 2002; aceito: 15 de abril de 2004)

\begin{abstract}
Anatomy of Tillandsia L. (Bromeliaceae) scapes from the "Campos Gerais" of Paraná, Brazil). The anatomy of Tillandsia crocata (E. Morren) Baker, T. gardneri Lindl., T. geminiflora Brongn., T. linearis Vell., T. lorentziana Griseb., T. mallemontii Glaziou ex Mez, T. recurvata L., T. streptocarpa Baker, T. stricta Soland ex Sims, T. tenuifolia L., and Tillandsia sp. scapes were studied. They occur in the "Campos Gerais", state of Paraná, southern Brazil. They are epiphytics on trunks or rock slopes. Scapes are both bent or straight revested by bracts and they grow up from the center of the foliar rosette. There are two patterns of distribution concerning the vascular bundles, one of them the peripherical bundles are condensed in the vascular cilyndre surrounded and/or immersed in a sclerotic cilyndre, the pericycle. The other presents vascular bundles scattered but concentred on the vascular cylindre periphery, total or partially involved in a fibrous sheath without a sclerotic cylinder. Most of these species presents scapes with thickened wall hypodermic and epidermic cells, and colateral vascular bundles that are total ou partially involved by fibers. The presence of thickened wall cells, hypodermis, raphids idioblasts, mucilage and air channel are adaptatives characters related to the epiphytic habit. The kind of thickness of the epidermic cells, hypodermis and pericycle; the distribution of vascular bundles, and the presence of composite vascular bundles could help species delimitation into the genus. It is presented a identification key of Tillandsia to the anatomic features of the scape.
\end{abstract}

Key words - anatomy, Bromeliaceae, scape, Tillandsia

RESUMO - (Anatomia de escapos de Tillandsia L. (Bromeliaceae) dos Campos Gerais do Paraná, Brasil). Estudou-se a anatomia dos escapos de Tillandsia crocata (E. Morren) Baker, T. gardneri Lindl., T. geminiflora Brongn., T. linearis Vell., T. lorentziana Griseb., T. mallemontii Glaziou ex Mez, T. recurvata L., T. streptocarpa Baker, T. stricta Soland ex Sims, T. tenuifolia L. e Tillandsia sp., que ocorrem nos Campos Gerais do Paraná. São plantas epífitas de galhos de árvores ou de paredões rochosos. Os escapos são eretos ou recurvados, revestidos por brácteas e emergem do centro da roseta foliar. Verificaram-se dois padrões anatômicos de distribuição dos feixes vasculares. Um padrão onde os feixes vasculares ocorrem condensados perifericamente no cilindro vascular, circundados e/ou imersos num cilindro esclerótico - periciclo. Outro padrão onde os feixes vasculares ocorrem dispersos, mas concentrados na periferia do cilindro vascular, envolvidos total ou parcialmente por bainha fibrosa, sem formar um cilindro esclerótico. A grande maioria das espécies apresenta escapos com epiderme de células espessadas, hipoderme com células espessadas, e feixes vasculares colaterais envolvidos total ou parcialmente por fibras. A presença de células com paredes espessadas, de hipoderme, de idioblastos de ráfides, mucilagem e canais de ar constituem características adaptativas ao hábito epifítico. Os tipos de espessamento das células epidérmicas, da hipoderme, e do periciclo, a distribuição dos feixes vasculares, e a presença de feixes vasculares compostos possibilitam separar as espécies dentro do gênero. É apresentada uma chave de identificação das Tillandsia estudadas com base nas características anatômicas do escapo.

Palavras-chave - anatomia, Bromeliaceae, escapos, Tillandsia

\section{Introdução}

Bromeliaceae apresenta distribuição tropical e subtropical, com aproximadamente 45 gêneros e cerca de 2.000 espécies (Cronquist 1981). É constituída por três subfamílias: Pitcairnioideae, Tillandsioideae e

\footnotetext{
1. Parte da tese de doutorado de Simone Segecin, Universidade Estadual Paulista, Rio Claro, São Paulo.

2. Rua República do Líbano n. 404, Jardim Carvalho, 84015-710 Ponta Grossa, Paraná, Brasil.

3. Universidade Estadual Paulista, Instituto de Biociências, Caixa Postal 199, 13506-900 Rio Claro, São Paulo, Brasil.

4. Autor para correspondência: rsmoro@uepg.br
}

Bromelioideae, separadas por caracteres florais, hábito e morfologia de frutos e sementes (Smith \& Downs 1974, 1977, 1979). Tillandsioideae caracteriza-se por apresentar plantas principalmente epífitas, sementes com apêndices plumosos, folhas com margens inteiras e limbo recoberto por escamas (Smith \& Downs 1977).

Tillandsia é o gênero que apresenta maior número de espécies, com cerca de 400, dentre os outros seis gêneros da subfamília (Cronquist 1981). No Brasil, na região dos Campos Gerais, PR, as espécies estão amplamente distribuídas, ocorrendo sobre rochas, paredões rochosos e matas ciliares.

Estudos anatômicos sobre escapos florais de Bromeliaceae foram realizados por Tomlinson (1969), 
a partir dos resultados de Mez (1896 apud Tomlinson 1969) e Solereder \& Meyer (1929 apud Tomlinson 1969). Esse estudo se restringiu à organização e distribuição dos feixes vasculares.

Tendo em vista a importância de estudos anatômicos de escapos de Bromeliaceae, pretende-se neste trabalho caracterizar a anatomia dos escapos de Tillandsia que ocorrem nos Campos Gerais, PR. Juntamente com isso, levantar as características que poderão auxiliar na taxonomia do grupo, bem como interpretar as possíveis adaptações ao ambiente.

\section{Material e métodos}

As amostras de escapos, em número de cinqüenta e cinco, de espécies de Tillandsia foram coletadas na região dos Campos Gerais, PR nas áreas de preservação ambiental, nos Parques Estaduais de Vila Velha e Guartelá e no Parque Municipal São Jorge. As exsicatas estão depositadas nos herbários da Universidade Estadual de Ponta Grossa (HUEPG) e no Herbarium Rioclarense (HRCB). Tillandsia crocata (E. Morren) Baker. BRASIL. PARANá: Ponta Grossa, 19-X-1997, S. Segecin 1 (HRCB); BRASIL. Paraná: Ponta Grossa, 19-XII-1997, S. Segecin 2 (HUEPG). Tillandsia gardneri Lindl. BRASIL. Paraná: Ponta Grossa, 6-I-1998, S. Segecin 3 (HRCB); BRASIL. Paraná: Ponta Grossa, 6-I-1998, S. Segecin 4 (HUEPG). Tillandsia geminiflora Brongn. BRASIL. PARANÁ: Ponta Grossa, 10-X-1999, S. Segecin 5 (HRCB); BRASIL. PARANÁ: Ponta Grossa, 10-XII-1999, S. Segecin 6 (HUEPG). Tillandsia linearis Vell. BRASIL. Paraná: Ponta Grossa, 9-I-1998, S. Segecin 7 (HRCB); BRASIL. PARANÁ: Ponta Grossa, 9-I-1998, S. Segecin 8 (HUEPG). Tillandsia lorentziana Griseb. BRASIL. PARANÁ: Ponta Grossa, 9-I-1998, S. Segecin 9 (HRCB); BRASIL. Paraná: Ponta Grossa, 9-I-1998, S. Segecin 10 (HUEPG). Tillandsia mallemontii Glaziou ex Mez. BRASIL. Paraná: Ponta Grossa, 12-II-1999, S. Segecin 11 (HRCB); BRASIL. PARANá: Ponta Grossa, 12-II-1999, S. Segecin 12 (HUEPG). Tillandsia recurvata L. BRASIL. Paraná: Ponta Grossa, 5-X-1997, S. Segecin 13 (HRCB); BRASIL. Paraná: Ponta Grossa, 5-X-1997, S. Segecin 14 (HUEPG). Tillandsia streptocarpa Baker. BRASIL. PARANá: Ponta Grossa, 12-XI-1998, S. Segecin 15 (HRCB); BRASIL. Paraná: Ponta Grossa, 12-XI-1998, S. Segecin 16 (HRCB). Tillandsia stricta Soland ex Sims. BRASIL. Paraná: Ponta Grossa, 6-I-1998, S. Segecin 17 (HRCB); BRASIL. PARANÁ: Ponta Grossa, 6-I-1998, S. Segecin 18 (HUEPG). Tillandsia tenuifolia L. BRASIL. PARANÁ: Ponta Grossa, 9-VIV-1998, S. Segecin 19 (HRCB); BRASIL. Paraná: Ponta Grossa, 9-VIV1998, S. Segecin 20 (HUEPG). Tillandsia sp. BRASIL. Paraná: Ponta Grossa, 2-X-1998, S. Segecin 21 (HRCB); BRASIL. Paraná: Tibagi, 2-X-1999, S. Segecin 22 (HUEPG).

Do material coletado, parte foi herborizada e parte foi fixada em FAA 50 (Johansen 1940) e, posteriormente, estocada em álcool etílico a $70 \%$ para os estudos anatômicos. Para análise anatômica, foram feitas secções à mão livre com lâminas de barbear, na região mediana dos escapos de pelo menos três indivíduos diferentes. As secções foram submetidas ao processo de dupla coloração com fucsina básica e azul de astra (Roeser 1962) e montadas em gelatina glicerinada.

Algumas secções do material fresco foram utilizadas para os testes microquímicos. Foram empregados reagente de Lugol (Bucherl 1962) e cloreto de zinco iodado (Strasburger 1911) para identificar o amido; Sudam III e Sudam IV (Johansen 1940) para as gotículas lipídicas; solução de floroglucina em meio ácido (Johansen 1940) para lignina; azul de astra (Roeser 1962) para celulose e solução de azul de metileno (Langeron 1949) para verificar a presença de mucilagem.

\section{Resultados}

Os escapos das Tillandsia estudadas sustentam inflorescências em cacho. Apresentam forma cilíndrica, emergindo-se da porção terminal da roseta foliar. Externamente são recobertos por brácteas em toda a sua extensão como pode ser observado em $T$. crocata (figura 1), T. lorentziana (figura 2), T. gardneri (figura 3) e T. tenuifolia (figura 4).

A distribuição espacial dos tecidos dos escapos das Tillandsia estudadas, está representada nos diagramas das secções transversais da região mediana dos mesmos (figuras 5-15). Apresentam forma cilíndrica, com epiderme, córtex e cilindro vascular distintos.

É possível agrupar os escapos em dois padrões principais de acordo com a estrutura do periciclo e a distribuição dos feixes vasculares segundo Mez (1896 apud Tomlinson 1969). O primeiro padrão é constituído por feixes vasculares isolados e espalhados (distribuição homogênea), estando os feixes vasculares periféricos mais próximos uns dos outros e com periciclo mais desenvolvido em relação aos feixes vasculares centrais, sem formar um cilindro pericíclico contínuo. Nesse padrão incluem-se Tillandsia gardneri (figura 6), T. geminiflora (figura 7), T. lorentziana (figura 9), T. stricta (figura 13) e T. tenuifolia (figura 14). No segundo padrão os feixes vasculares da periferia do cilindro vascular estão concentrados e imersos num cilindro esclerótico (periciclo fibroso) que delimita o córtex e é contínuo. Os feixes vasculares centrais apresentam periciclo menos desenvolvido e são em menor número. Incluem-se nesse padrão: Tillandsia crocata (figura 5), T. linearis (figura 8), T. mallemontii (figura 10), T. recurvata (figura 11), T. streptocarpa (figura 12) e Tillandsia sp. (figura 15).

Algumas Tillandsia apresentam feixes vasculares colaterais compostos, como pode ser observado em 
T. lorentziana (figuras 9, 22) e T. mallemontii (figura 24).

Nas espécies de Tillandsia estudadas, os feixes vasculares são colaterais, envolvidos pela endoderme e pelas fibras pericíclicas. Estão concentrados na periferia do cilindro vascular em $T$. crocata (figura 5), $T$. linearis (figura 8), T. mallemontii (figura 10), T. recurvata (figura 11) e T. streptocarpa (figura 12). Em T. gardneri (figura 6), T. geminiflora (figura 7), T. lorentziana (figura 9), T. stricta (figura 13), T. tenuifolia (figura 14) e Tillandsia sp. (figura 15) os feixes vasculares estão distribuídos homogeneamente no cilindro vascular, sem a presença de um anel pericíclico contínuo de paredes espessas e muitas camadas de células delimitando a
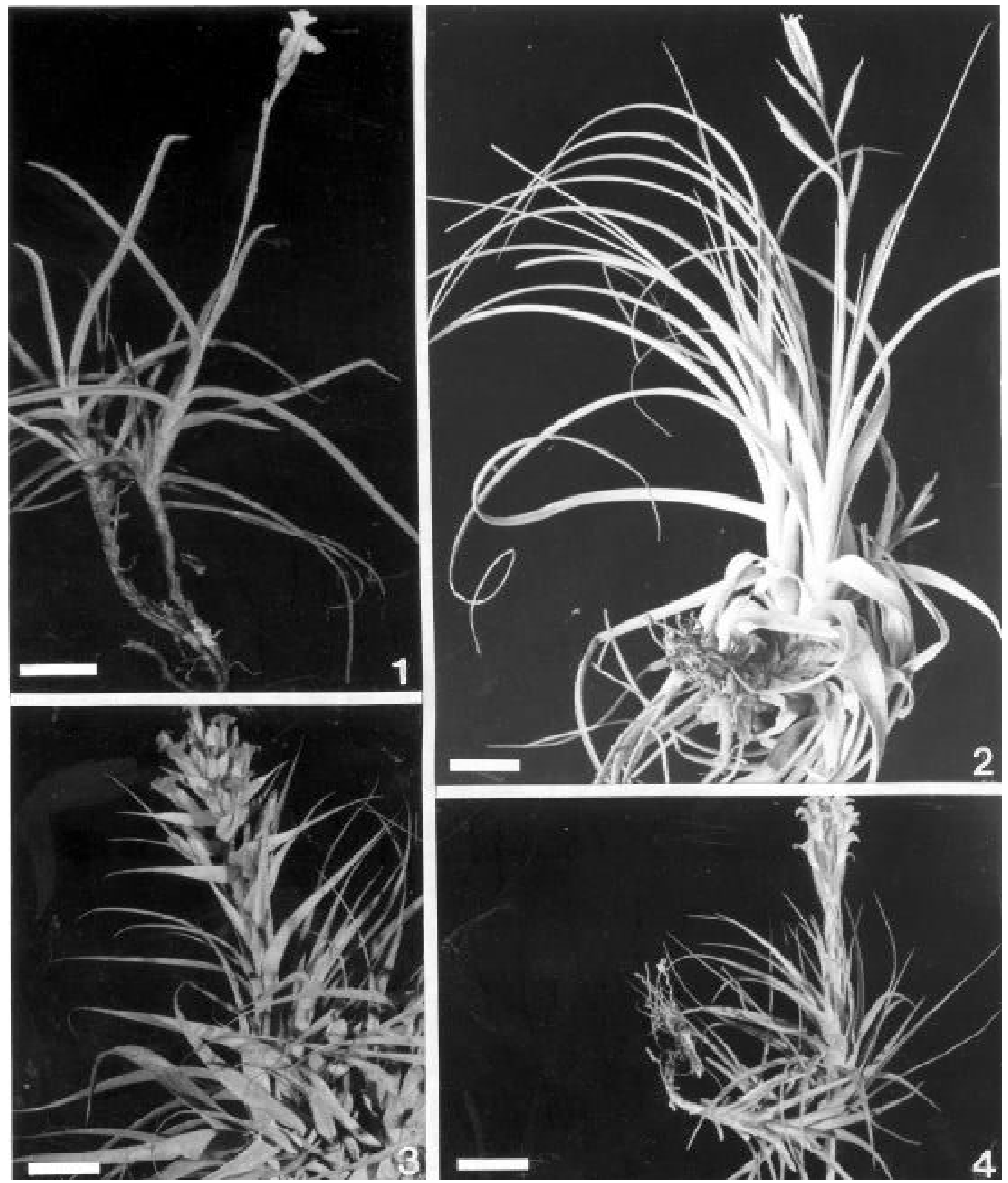

Figuras 1-4. Aspectos morfológicos de Tillandsia: 1 . T. crocata; 2. T. lorentziana; 3. T. gardneri; 4 . T. tenuifolia . Barras $=2 \mathrm{~cm}$ $(1) ; 3,5 \mathrm{~cm}(2) ; 3 \mathrm{~cm}(3) ; 2,2 \mathrm{~cm}(4)$.

Figures 1-4. Morphological aspects of Tillandsia: 1. T. crocata; 2. T. lorentziana; 3. T. gardneri; 4 . T. tenuifolia . Bars $=2 \mathrm{~cm}$ (1); $3.5 \mathrm{~cm}(2) ; 3 \mathrm{~cm}(3) ; 2.2 \mathrm{~cm}(4)$. 


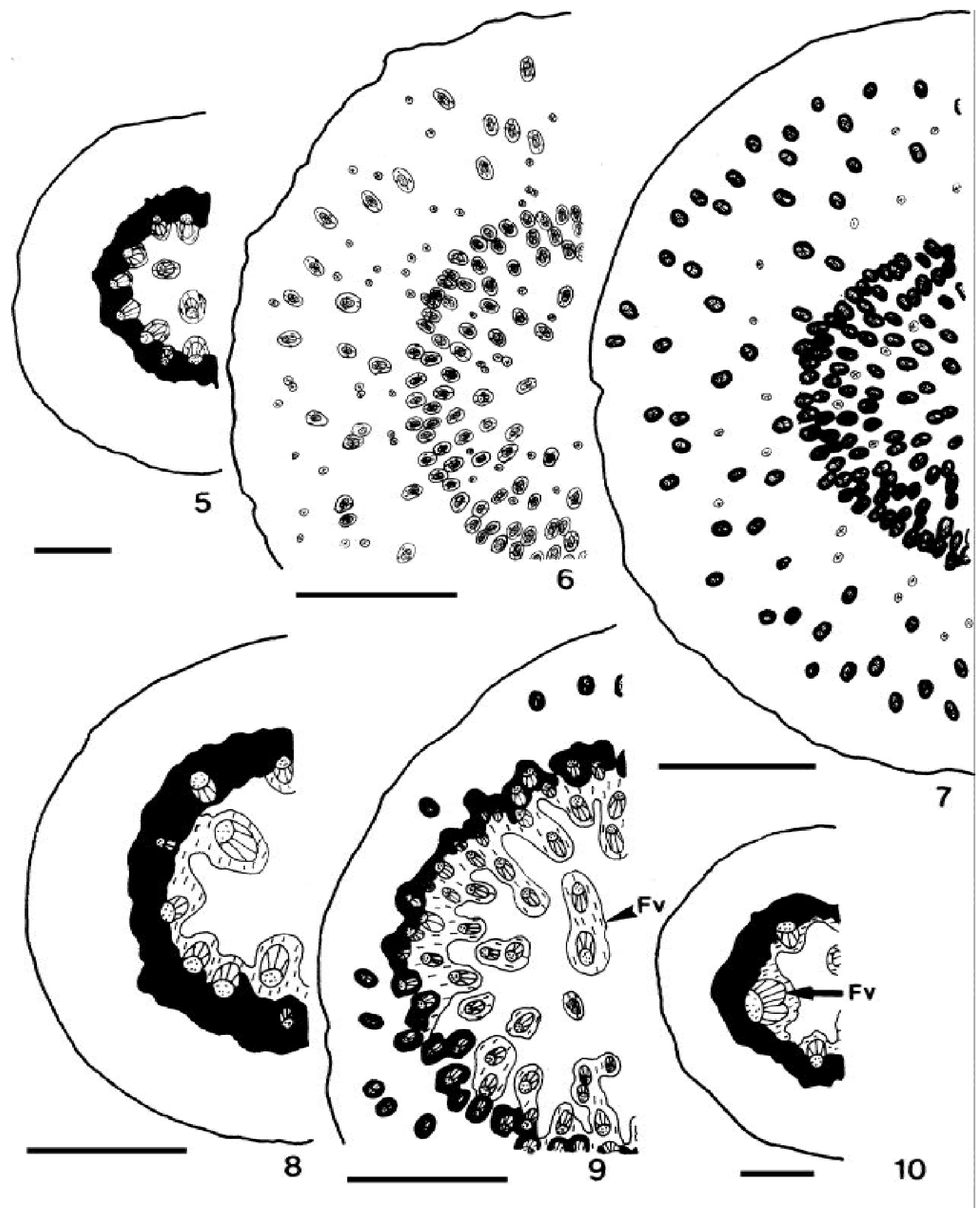

Figuras 5-10. Diagramas de secções transversais da região mediana de escapos. 5. Tillandsia crocata. 6. T. gardneri. 7. T. geminiflora. 8. T. linearis. 9. T. lorentziana. 10. T. mallemontii. Barras $=300 \mu \mathrm{m}(5,8) ; 600 \mu \mathrm{m}(6,7,9) ; 100 \mu \mathrm{m}(10)$. $(\mathrm{Fv}=$ feixes vasculares $)$.

Figures 5-10. Diagrams of transversal sections of median region of scapes. 5. Tillandsia crocata. 6. T. gardneri. 7. T. geminiflora. 8. T. linearis. 9. T. lorentziana. 10. T. mallemontii. Bars $=300 \mu \mathrm{m}(5,8) ; 600 \mu \mathrm{m}(6,7,9) ; 100 \mu \mathrm{m}(10)$. (Fv = vascular bundles). 


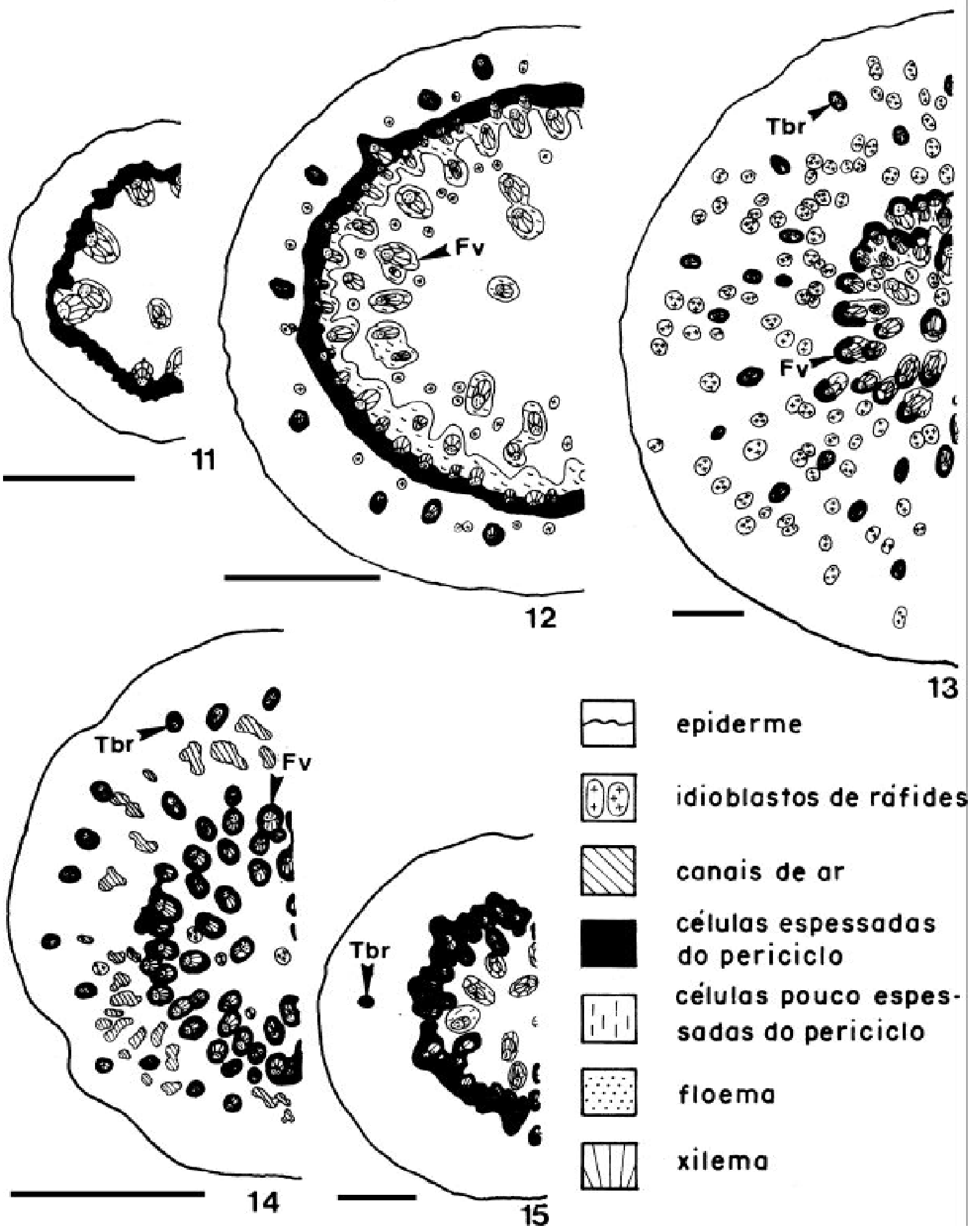

Figuras 11-15. Diagramas de secções transversais da região mediana de escapos: 11. Tillandsia recurvata. 12. T. streptocarpa. 13. T. stricta. 14 . T. tenuifolia. 15. Tillandsia sp. Barras $=300 \mu \mathrm{m}(11,13-15) ; 600 \mu \mathrm{m}(12,14) .(\mathrm{Fv}=$ feixes vasculares; $\mathrm{Tbr}=$ traço de bráctea).

Figures 11-15. Diagrams of transversal sections of median region of scapes: 11. Tillandsia recurvata. 12. T. streptocarpa. 13. T. stricta. 14. T. tenuifolia. 15. Tillandsia sp. Bars $=300 \mu \mathrm{m}(11,13-15) ; 600 \mu \mathrm{m}(12,14) .(\mathrm{Fv}=\mathrm{vascular}$ bundles; $\mathrm{Tbr}=\mathrm{trace}$ of bract). 
região cortical da vascular.

Em Tillandsia streptocarpa (figura 12), T. stricta (figura 13), T. tenuifolia (figura 14), T. lorentziana (figura 22), T. mallemontii (figura 24) e T. recurvata (figura 25), ocorrem feixes vasculares colaterais compostos, variando quanto à sua estrutura e localização no cilindro vascular. Em T. stricta e T. mallemontii, geralmente, os feixes vasculares compostos possuem bainha esclerenquimática comum, situados na periferia do cilindro vascular, como pode ser observado na figura 24. Em T. recurvata (figura 25), os feixes vasculares compostos não apresentam a bainha esclerenquimática comum, mas também ocorrem na periferia do cilindro vascular. Em Tillandsia tenuifolia (figura 14), os feixes vasculares compostos apresentam bainha esclerenquimática comum e ocorrem, geralmente, na medula do cilindro vascular. Em T. lorentziana (figura 22) os feixes vasculares compostos se encontram um em frente ao outro, com bainha esclerenquimática individualizada e, também, ocorrem na medula do cilindro vascular.

Na região cortical dos entrenós ocorrem traços vasculares das brácteas, como podem ser observados em Tillandsia stricta (figuras 13, 27), T. tenuifolia (figuras 14, 29) e Tillandsia sp. (figuras 15, 31). Observam-se canais de ar apenas em T. tenuifolia (figuras 14, 29). Na grande maioria das Tillandsia estudadas foi observada a ocorrência de idioblastos com ráfides, como observado em T. gardneri (figura 18). Em algumas figuras indicadas se observa apenas o idioblasto vazio, pois as ráfides saem da célula que fica com a parede celular rompida por causa da preparação do material, como observado em T. streptocarpa (figura 26), T. stricta (figura 28) e Tillandsia sp. (figura 31). Em T. streptocarpa os idioblastos com ráfides são grandes, apresentam forma arredondada e encontram-se em conjunto (figura 26), nas demais espécies estudadas os idioblastos com ráfides geralmente são menores e podem ocorrer isolados (figuras 6-7, 12-14, 28, 31). Na maioria das espécies foi observada mucilagem nas células corticais (figuras 19, 29).

A epiderme do escapo das espécies de Tillandsia estudadas é uniestratificada, com células de tamanho reduzido quando comparadas às células corticais; apresentam a forma de arredondada a lenticular, em secção transversal, com cutícula de espessura variável (figuras 16-17, 19, 21, 23-25, 27, 29-30). As paredes das células epidérmicas são levemente espessadas por celulose em T. gardneri e T. geminiflora (figuras 17, 19, respectivamente). Nas demais Tillandsia estudadas as células epidérmicas apresentam-se com as paredes periclinais externas finas e as demais paredes espessadas e lignificadas (figuras 16, 21, 23-25, 27, 29-30). Em T. crocata o espessamento das paredes anticlinais e periclinal interna formam uma figura de cunha (figura 16), em T. linearis o espessamento dessas paredes formam uma meia lua (figura 23), nas demais espécies estudadas o espessamento dessas paredes é homogêneo variando com o estágio de desenvolvimento do escapo.

O córtex, em secção transversal, apresenta células parenquimáticas de forma variada, desde arredondadas a hexagonais, paredes finas, mucilaginosas e com espaços intercelulares conspícuos, como em Tillandsia crocata (figura 16-seta). A camada externa do córtex, situada logo abaixo da epiderme, constitui a hipoderme e se apresenta espessada e lignificada em $T$. lorentziana (figura 21), T. streptocarpa e Tillandsia sp. (figura 30). Nas demais espécies estudadas essa camada celular se apresenta com paredes finas, como em $T$. gardneri e T. mallemontii (figuras 17, 24, respectivamente), e levemente espessadas, como em $T$. crocata, T. geminiflora, T. linearis, T. recurvata, T. stricta, e T. tenuifolia (figuras 16, 19, 23, 25, 27, 29, respectivamente).

A endoderme é constituída por uma camada de células arredondadas com paredes finas, como se observa em $T$. crocata (figura 16). Pode se apresentar descontínua como em T. gardneri (figura 18) e T. tenuifolia. Nas demais Tillandsia apresenta-se contínua, como em $T$. crocata (figura 16) e T. geminiflora (figura 20).

O cilindro vascular é delimitado pelo periciclo que se apresenta constituído por várias camadas de células de paredes espessadas e lignificadas, como em Tillandsia lorentziana (figura 9), T. crocata (figura 16), T. linearis (figura 23), T. mallemontii (figura 24), T. recurvata (figura 25) e T. streptocarpa (figura 26). Por outro lado, o cilindro vascular pode estar constituído por uma a três camadas celulares, como em $T$. stricta (figura 28), T. tenuifolia, T. geminiflora (figura 20) e Tillandsia sp. (figura 31), ou por células com paredes pouco espessadas, como em T. gardneri (figura 18 - setas).

O periciclo se apresenta descontínuo, como em T. lorentziana (figura 9), T. tenuifolia (figura 14), T. gardneri (figura 18), T. stricta (figura 28) ou contínuo como em $T$. crocata (figura 16), T. geminiflora (figura 20), T. linearis (figura 23), T. mallemontii (figura 24), T. recurvata (figura 25), T. streptocarpa (figura 26) $\mathrm{e}$ Tillandsia sp. (figura 31). 

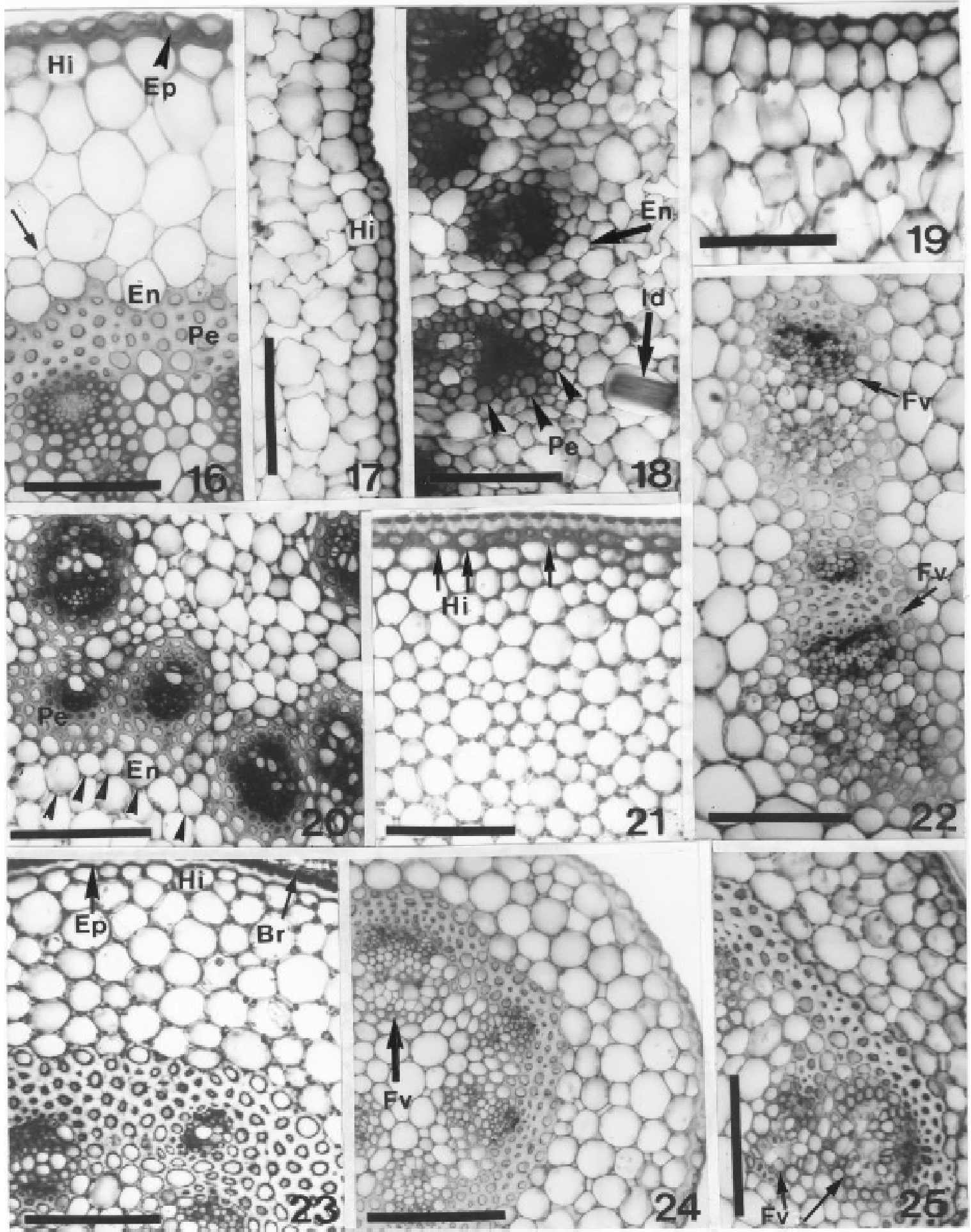

Figuras 16-25. Aspectos anatômicos de secções transversais da região mediana dos escapos: 16. Tillandsia crocata (seta = espaço intercelular). 17-18. T. gardneri. 19-20. T. geminiflora. 21-22. T. lorentziana. 23. T. linearis. 24. T. mallemontii. 25. T. recurvata . Barras $=100 \mu \mathrm{m}(16-25) .(\mathrm{Br}=$ bráctea $; \mathrm{En}=$ endoderme; $\mathrm{Ep}=$ epiderme; $\mathrm{Fv}=$ feixes vasculares; $\mathrm{Hi}=$ hipoderme; $\mathrm{Id}=$ idioblasto com ráfides; $\mathrm{Pe}=$ periciclo).

Figures 16-25. Anatomical aspects of transversal sections of median regions of scapes: 16. Tillandsia crocata $($ arrow = intercellular space). 17-18. T. gardneri. 19-20. T. geminiflora. 21-22. T. lorentziana. 23. T. linearis. 24. T. mallemontii. 25. T. recurvata. Bars $=100 \mu \mathrm{m}(16-25) .(\mathrm{Br}=$ bracts; En = endodermis; Ep = epidermis; Fv = vascular bundles; Hi = hypodermis; Id = idioblast wich raphides; $\mathrm{Pe}=$ pericycle). 
Chave de identificação das espécies de Tillandsia dos Campos Gerais, PR, utilizando dados anatômicos dos escapos

1. Epiderme com células de parede fina celulósica

2. Endoderme contínua

T. geminiflora

2. Endoderme descontínua T. gardneri

1. Epiderme com células de parede espessada lignificada

3. Hipoderme com células de paredes espessadas

4. Idioblastos com ráfides grandes e em conjunto

T. streptocarpa

4. Idioblastos com ráfides menores, isolados ou não

5. Presença de feixes vasculares compostos

T. lorentziana

5. Ausência de feixes vasculares compostos Tillandsia sp.

3. Hipoderme com células de paredes finas

6. Periciclo descontínuo, com poucas camadas e células de paredes pouco espessadas

7. Endoderme contínua; córtex sem canais de ar T. stricta

7. Endoderme descontínua; córtex com canais de ar.

T. tenuifolia

6. Periciclo contínuo, com muitas camadas de células de paredes espessadas

8. Presença de feixes vasculares compostos

9. Feixes vasculares compostos com periciclo comum

T. mallemontii

9. Feixes vasculares compostos com periciclo independente

T. recurvata

8. Presença de feixes vasculares simples.

10. Epiderme com espessamento em forma de cunha

T. crocata

10. Epiderme com espessamento em forma de meia lua

T. linearis

\section{Discussão}

As espécies de Tillandsia estudadas neste trabalho são epífitas, ocorrendo principalmente nas matas ciliares, sobre galhos de árvores ou crescendo sobre afloramentos rochosos.

De acordo com Nunes (1997), existe uma relação espécie substrato que pode ser muito específica nas Bromeliaceae. Em relação as espécies de Bromeliaceae estudadas observa-se claramente essa relação, principalmente quanto à tolerância da luz solar e estratégias anatômicas relacionadas a esta condição. Pittendrigh (1948), correlacionou a morfologia interna ao hábito de espécies de Bromeliaceae, e confirmou a relação hábito e tolerância à luz pela presença de estruturas anatômicas adaptadas a tal situação. Considerando-se a insolação direta, observa-se nas espécies de Bromeliaceae estudadas com hábito sobre afloramentos rochosos, onde a insolação, bem como a ação do vento é maior, que as células epidérmicas apresentam-se espessadas, como estratégia para evitar a transpiração excessiva, bem como suporte mecânico contra ação dos ventos, corroborando com as observações de Nunes (1997) e Pittendrigh (1948).

O estudo anatômico dos escapos de Tillandsia da região dos Campos Gerais, PR, permitiu verificar que as mesmas possuem características comuns ao gênero, reconhecidas por Solereder \& Meyer (1929 apud Tomlinson 1969). A presença de córtex e cilindro central distintos com sistema vascular organizado geralmente em anel, com feixes vasculares distribuídos mais regularmente, sem raízes intracorticais, são alguns dos caracteres comuns.

O espessamento das células da epiderme, da hipoderme em algumas espécies e das células pericíclicas tem como prováveis funções evitar altas temperaturas internas, diminuindo a evaporação de água dos tecidos, evitando assim o colapso das células pelo murchamento, bem como conferindo sustentação mecânica contra a ação dos ventos. Essas adaptações estão relacionadas à economia hídrica que está geralmente associada ao epifitismo e são características marcantes de plantas que vivem em ambientes estressantes, conforme observado por Krauss (1948), Pyykkö (1966), Esau (1977) e Brighigna et al. (1984).

Mez (1896 apud Tomlinson 1969) classificou os escapos de alguns representantes de Bromeliaceae de acordo com a distribuição dos feixes vasculares e da estrutura do periciclo em dois padrões principais. No primeiro padrão os feixes vasculares estão distribuídos homogeneamente; os periféricos com bainha fibrosa (periciclo), mais espessa em relação aos feixes centrais, não havendo continuidade do periciclo. Nesse padrão encaixam-se Tillandsia gardneri, T. geminiflora, 
T. stricta e T. tenuifolia estudadas neste trabalho. No segundo padrão os feixes vasculares se encontram concentrados na periferia do cilindro vascular e imersos num cilindro esclerótico (periciclo) contínuo que delimita o córtex; os feixes vasculares centrais estão em menor número. Nesse segundo padrão encaixam-se T. crocata, T. mallemontii, T. lorentziana, T. linearis, T. recurvata, T. streptocarpa e Tillandsia sp. estudadas no presente trabalho.

Observou-se, através dos resultados obtidos neste estudo, que a organização e a estrutura do cilindro vascular não constitui um caráter consistente para separar as espécies, apenas subdividindo o gênero em dois grandes grupos.

Canais de ar conspícuos foram observados apenas em Tillandsia tenuifolia, nas demais espécies estudadas estes são discretos. Segundo Meyer (1940), os canais de ar presentes no córtex interno das raízes teriam a função de transportar água por capilaridade. Considerando que as espécies de Tillandsia estudadas são epífitas atmosféricas e crescem sob constante estresse hídrico, essa função poderia ocorrer naquelas espécies que apresentam canais de ar no córtex do escapo. Possivelmente a presença de canais de ar, em

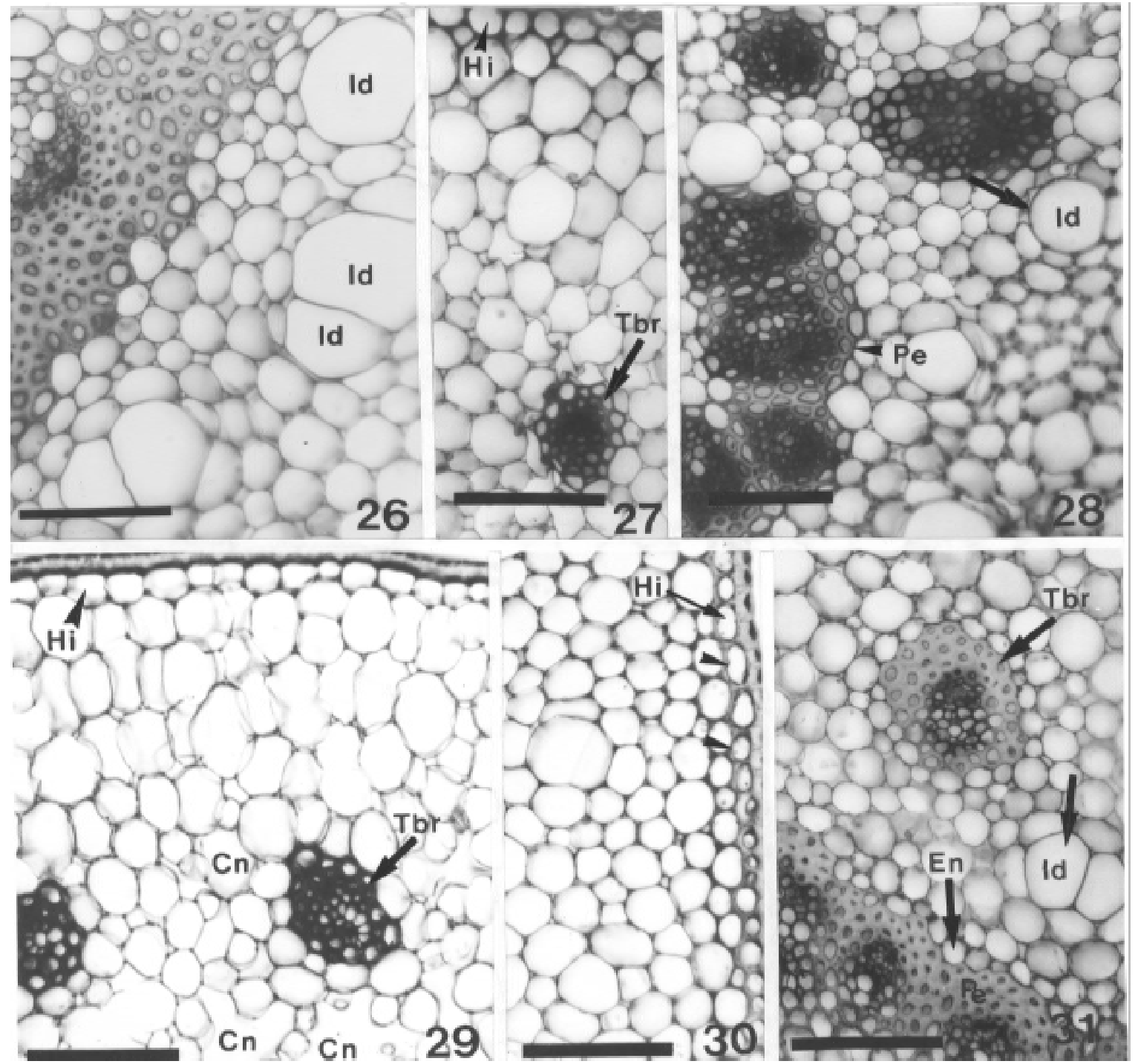

Figuras 26-31. Aspectos anatômicos de secções transversais da região mediana dos escapos: 26. Tillandsia streptocarpa. 27-28. T. stricta. 29. T. tenuifolia. 30-31. Tillandsia sp. Barras $=100 \mu \mathrm{m} .(\mathrm{Cn}=$ canal de ar; $\mathrm{En}=$ endoderme; $\mathrm{Hi}=$ hipoderme; $\mathrm{Id}=$ idioblasto; $\mathrm{Pe}=$ periciclo; $\mathrm{Tbr}=$ traço de bráctea) .

Figures 26-31. Anatomical aspects of transversal sections of median regions of scapes: 26. Tillandsia streptocarpa. 27-28. T. stricta. 29. T. tenuifolia. 30-31. Tillandsia sp. Bars $=100 \mu \mathrm{m} .(\mathrm{Cn}=$ air channel; $\mathrm{En}=$ endodermis; $\mathrm{Hi}=$ hypodermis; Id = Idioblast; $\mathrm{Pe}=$ pericycle; $\mathrm{Tbr}=$ trace of bract $)$. 
qualquer órgão vegetativo, esteja relacionada a essa função, como estratégia de transportar mais rapidamente água. Os canais de ar podem ser formados pelo aumento dos espaços intercelulares, causado pelo afastamento das células (esquizogenia), ou por vários graus de desintegração celular (lisigenia) (Smirnoff \& Crawford 1983). Nas espécies estudadas não se observaram restos de paredes celulares no córtex dos escapos, fato que sugere a esquizogenia.

De acordo com Costa (1975), em muitas monocotiledôneas, o ácido oxálico ocorre na forma de cristais de oxalato de cálcio insolúvel, apresentando-se de formas diversas, como na forma de ráfides em idioblastos. Segundo Brighigna et al. (1984), a ocorrência de idioblastos com ráfides, distribuídos no córtex, no mesofilo, e ao redor dos feixes vasculares em Bromeliaceae, está relacionada com a necessidade de neutralizar a grande quantidade de ácido oxálico produzido nas folhas. Nos escapos das Tillandsia estudadas os idioblastos com ráfides também se encontram ao redor dos feixes vasculares, sugerindo ação na neutralização do ácido oxálico, como nas folhas das espécies estudadas por Brighigna et al. (1984). Prichid \& Rudall (2000), também fazem referência aos cristais aciculares na prevenção contra ataque de herbívoros.

Juntamente com o espessamento das paredes das células e com a presença dos canais de ar, a mucilagem atua, também, como estratégia para economia hídrica. De acordo com Fahn \& Cutler (1992), a ocorrência de idioblastos com ráfides e mucilagem é considerada como adaptação das plantas às condições xerofíticas. A mucilagem é substância macromolecular, de natureza glicídica, que em presença da água intumesce e fica com aspecto viscoso. É considerada um produto normal do metabolismo de algumas plantas. Segundo Costa (1975), exerce a função de reserva, como substância alimentar e de retenção de água, constituindo-se assim, numa reserva útil. De acordo com Esau (1977), a presença de mucilagem nos tecidos, além de evitar a dessecação, também atua na troca iônica possibilitando a quelagem dos nutrientes. A presença de mucilagem nas espécies estudadas possivelmente está relacionada com o hábito epifítico e provavelmente é utilizada como substância de reserva energética e hídrica.

Analisando os resultados obtidos no presente trabalho, foram selecionados alguns caracteres anatômicos específicos que possibilitaram separar as espécies dentro do gênero e fornecer uma chave dicotômica de identificação para as espécies de Tillandsia dos Campos Gerais, Paraná. Características como: espessamento das paredes das células da epiderme, da hipoderme e do periciclo; estrutura da endoderme; distribuição dos feixes vasculares e presença de feixes vasculares compostos possibilitam separar as espécies dentro do gênero. A presença de células com paredes espessadas; idioblastos com ráfides e células com mucilagem, constituem características adaptativas ao hábito epifítico.

Agradecimentos - Ao Conselho Nacional de Desenvolvimento Científico e Tecnológico (CNPq) pela bolsa de Doutorado concedida à Simone Segecin e pela bolsa de Produtividade em Pesquisa concedida à Vera Lucia Scatena.

\section{Referências bibliográficas}

BRIGHIGNA, L., FIORDI, A. \& PALANDRI, M.R. 1984. Structural characteristcs of mesophyll in some Tillandsia species. Phytomorphology 34:191-200.

BÜCHERL, W. 1962. Técnica microscópica. Polígono, São Paulo.

COSTA, A.F. 1975. Farmacognosia. Fundação Calouste Gulbenkian, Lisboa.

CRONQUIST, A. 1981. An integrated system of classification of flowering plants. Columbia University Press, New York.

ESAU, K. 1977. Anatomy of seed plants. John Wiley \& Sons, London.

FAHN, A. \& CUTLER, D.F. 1992. Xerophytes. Encyclopedia of plant taxonomy. Gebrüder Borntraeger, Berlin.

JOHANSEN, D.A. 1940. Plant microtechinique. MacGraw-Hill. New York.

KRAUSS, B.H. 1948. Anatomy of the vegetative organs of the Pineapple, Annanas comosus (L.) Merr. Botanical Gazette 110:159-217.

LANGERON, M. 1949. Précis de Microscopie. Masson et Ciencie, Paris.

MEYER, L. 1940. Zur anatomie und entwicklungsgeschichte der Bromeliaceenwurzeln. Planta 31: 492-522.

NUNES, J.V. C. 1997. Estudo florístico e fenomorfológico de Tillandsioideae - Bromeliaceae na Serra do Cipó, MG. Dissertação de mestrado, Universidade de São Paulo, São Paulo.

PITTENDRIGH, C.S. 1948. The bromeliad - anopheles-malaria complex in Trinidad. I. The bromeliad flora. Evolution 2:58-89.

PRYCHID, C.J. \& RUDALL, P.J. 2000. Distribution of calcium oxalate crystals in monocotyledons. In Monocots: systematics and evolution (K.L. Wilson \& D.A. Morrison, eds.). CSIRO, Collingwood, p.159-161.

PYYKKÖ, M. 1966. The leaf anatomy of east Patagonian xeromorphic plants. American Journal of Botany 68:64-71.

ROESER, K.R. 1962. Die nadel der schwarzkiefer-masenprodukt und keinstwert der natur. Microkosmos 61:33-36. 
SMIRNOFF, N. \& CRAWFORD, R.M.M. 1983. Variation in the structure and response to flooding root aerenchyma in some wetland plants. Annals of Botany 51:237-249.

SMITH, L.B. \& DOWNS, R.J. 1974. Pitcairnioideae (Bromeliaceae). Flora Neotropica Monographies 4:1-658.

SMITH, L.B. \& DOWNS, R.J. 1977. Tillandsioideae (Bromeliaceae). Flora Neotropica Monographies 4:663-1492.
SMITH, L.B. \& DOWNS, R.J. 1979. Bromelioideae (Bromeliaceae). Flora Neotropica Monographies 14:1604-1724.

STRASBURGER, E. 1911. Handbook of practical botany. George Allen, London.

TOMLINSON, P.B. 1969. III - Commelinales-Zingiberales. In Anatomy of the Monocotyledons. (C.R. Metcalf, ed.). Clarendon Press, Oxford, p.1-446. 
